\title{
Development of a Model for High Precursor Conversion Efficiency Pulsed- Pressure Chemical Vapor Deposition (PP-CVD) Processing
}

\author{
Hadley M. Cave, Susan P. Krumdieck* \& Mark C. Jermy \\ Department of Mechanical Engineering, University of Canterbury, Private Bag 4800, Christchurch 8020, New Zealand.
}

\begin{abstract}
A model of the movement of precursor particles in the unsteady Pulsed-Pressure Chemical Vapour Deposition (PP-CVD) process is developed to study the high conversion efficiencies observed experimentally in this process. Verification of the modelling procedures was conducted through a study of velocity persistence in an equilibrium gas and through Direct Simulation Monte Carlo (DSMC) simulations of unsteady self-diffusion processes. The model results demonstrate that in the PP-CVD process the arrival time for precursor particles at the deposition surface is much less than the reactor pump-down time, resulting in high precursor conversion efficiencies. Higher conversion efficiency was found to correlate with smaller size solvent molecules and moderate reactor peak pressure.
\end{abstract}

Keywords: Pulsed pressure chemical vapour deposition (PP-CVD), Precursor conversion efficiency, Diffusion, DSMC, Process modelling.

"Corresponding author. Tel.: +64 33642987 ext 7248; fax: +64 3364 2078, e-mail: susan.krumdieck@canterbury.ac.nz.

\section{Introduction}

Over the past several decades, chemical vapour deposition (CVD) has become an increasingly important process for the manufacture of thin film materials. These materials have importance in such diverse applications as electronics, bio-technology and optics. As 
thin film technology has advanced, new types of CVD processes have been developed to manufacture these films [1].

The motivation for this work is the patented Pulsed-Pressure CVD (PP-CVD) process developed by Raj et al. [2]. Like other CVD processes deposition occurs via the thermal decomposition of the precursor on a heated substrate, however unlike other processes the precursor is delivered in an unsteady manner whereby timed pulses of precursor are released into a continuously evacuated reactor volume. The technique has been used successfully to deposit films from metal-organic precursors including titania films from titanium isopropoxide (TTIP) dissolved in toluene and delivered into the reactor as a liquid via an ultrasonic nozzle [3]. Yttria-stabilised zirconia (YSZ) has also been deposited using the same technique $[4,5]$.

Figure 1 shows a functional schematic of a PP-CVD reactor, along with a plot of the reactor pressure during operation. For gaseous precursors, during the injection phase $(0<t<$ $t_{i}$ ) a solenoid valve is opened which releases the precursor in the high pressure source vessel into the reactor volume at pressure $P_{\min }$. When the valve closes at $t=t_{i}$ the reactor is at its maximum pressure of $P_{\max }$. During the pump-down period $\left(t_{i}<t<t_{p}\right)$ the reactor is evacuated by the vacuum pump and the reactor pressure during this phase is given by:

$$
P(t)=P_{\text {min }}+\left(P_{\text {max }}-P_{\text {min }}\right) \exp \left(-\frac{t}{\tau_{R}}\right)
$$

where $\tau_{\mathrm{R}}$ is the reactor pump-down constant which can be determined experimentally by fitting the exponential function to the measured pressure profile [6].

Experimental studies and a phenomenological model of PP-CVD by Krumdieck and Raj have reported that during the deposition of titania, the conversion efficiency of the TTIP precursor into solid film exceeds $90 \%$ under certain operating conditions $[3,7,8]$. Efficiencies this high have not been reported in conventional CVD processing. It was 
proposed by Krumdieck et al. that during the injection phase there is a mass transport regime in which expansion effects dominate over continuum effects [9] and a study of the injection phase using computational fluid dynamics (CFD) by Cave et al. showed that this continuum breakdown region sweeps through the reactor volume resulting in a highly uniform mass flux field at the beginning of the pump-down phase [10].

At the low reactor pressures which occur during PP-CVD, or where the scale of macroscopic property gradients is of the order of the mean free path, the continuum description of gas dynamics becomes invalid. In this rarefied state, a molecular description of the reactor flow is required. Several methods exist for simulating rarefied flow including molecular dynamics (MD) simulations, where molecular motion and collisions are calculated deterministically, and the Lattice-Boltzmann method (LBM), which solves a simplified version of the Boltzmann equation over a grid [11]. However, the proceeding methods suffer respectively from enormous computational expense and poor physical reality, so the preferred technique for modelling rarefied flows has become the Direct Simulation Monte Carlo (DSMC) technique [12]. Here the movement and collisions of a large number of test particles are decoupled over a time step which is a fraction of the mean collision time. The technique is well established and has been used to simulate flow applications as diverse as high altitude aerodynamics [13], micro-thrusters for space applications [14] and steady flow CVD applications [15].

Modelling of the PP-CVD process using DSMC presents some significant challenges. The wide range of gas densities experienced and the unsteady nature of the flow means that modelling the entire process using the DSMC technique requires significant computational expense, even given the $2 \mathrm{D}$ axisymmetric nature of the flow. This problem is exacerbated by the relatively large ratio of solvent molecules to precursor molecules meaning a large number of particles must be simulated to maintain accuracy in precursor concentrations. A technique 
used to overcome a similar problem is the stochastic biatomic collision theory (BCT) method developed by Groves to model the Directed Vapour Deposition (DVD) of copper [16]. Here the paths of a large number of reactant molecules are tracked individually through a steady background gas field generated using DSMC. The technique assumes the concentration of reactant molecules is low enough that there is negligible interaction between them and utilises a complex method for the determination of vapour atom mean free path and collision dynamics.

\section{Reactor Efficiency Model}

\subsection{Model Structure}

The reactor efficiency model used in this study was loosely based on the BCT method, however mean free path calculations and the interaction of molecules during collision events were simulated using either the hard sphere (HS) collision model or the variable soft sphere (VSS) model developed by Koura and Matsumoto [17]. In this way the movement of the test particles through the reactor volume was decoupled from collision events in much the same way as the DSMC method, while allowing the use of readily available collision parameters. The computational flow of the model, known as the Pulsed Injection Efficiency Simulation (or "PIES") model, is illustrated in figure 2 . Here $\mathrm{N}_{\text {parts }}$ represents the number of test particles tracked for each simulation run.

\subsection{Model Development}

As shown by Bird [12], the vast majority of collisions in a dilute gas involve only two atoms. Furthermore, for these simulations it was assumed collisions were elastic such that there was no exchange of energy between the participant molecule's translational and internal 
degrees of freedom. In binary elastic collisions momentum and energy are conserved and the transport mean free path is given by [18]:

$$
\lambda_{m}(t)=\frac{R T}{\sqrt{2} P(t) N_{A} \sigma_{M}}
$$

where $R$ is the universal gas constant $(8.314 \mathrm{~kJ} / \mathrm{kmolK}), N_{A}$ is Avogadro's number $\left(6.023 \times 10^{23}\right.$ molecules $\left./ \mathrm{mol}\right)$ and $\sigma_{M}$ is the momentum transfer collision cross section.

The actual free path of a molecule is distributed about the mean value given in equation (2) with the probability of a particle travelling at least a distance $x$ before a collision being $e^{-x / \lambda_{m}}[18]$, hence the probability of a collision occurring between $x$ and $x+d x$ is [16]:

$$
\frac{1}{\lambda_{m}} e^{-x / \lambda_{m}} d x
$$

The momentum transfer collision cross section, which occurs in the Chapman-Enskog expression for diffusion coefficients [19], is given by Bird [12] as:

$$
\sigma_{M}=2 \pi \int_{0}^{\pi}(1-\cos \chi) \sigma(\chi) \sin \chi d \chi
$$

where $\chi$ is the deflection angle of the relative velocities of either molecule during collision and $\sigma(\chi)$ is the angular cross section which can be expressed in terms of an impact parameter $b$ which defines the distance of closest approach between the undisturbed trajectories of the collision partners:

$$
\sigma(\chi)=\frac{b}{\sin \chi}\left|\frac{d b}{d \chi}\right|
$$

Substitution of equation (5) into (4) and invoking the change in variable gives:

$$
\sigma_{M}=2 \pi \int_{0}^{b_{\max }}(1-\cos \chi) b d b
$$

To define the momentum cross section, and hence the transport mean free path, a relationship must be determined between the collision parameters $b$ and $\chi$, which are functions 
of the energy of the collision event. In the implementation of the BCT model by Groves, a relatively complex iterative procedure is employed to determine these parameters for the high energy copper molecules. However, here either the phenomenological hard sphere or VSS models are used primarily because of their relative simplicity and due to the ready availability of input parameters.

For the hard sphere model it can be shown that the momentum cross section is equivalent to the total collision cross section $\sigma_{M}=\sigma_{T}=\pi d_{12}^{2}$ where $d_{12}$ is the mean of the hard sphere diameter of the two collision partners [12]. The primary weakness of the hard sphere model is that in real gases the total collision cross section is a function of the relative translational energy of the collision partners and so is a function of temperature. The variable hard sphere (VHS) model [20], in which the hard sphere diameter becomes a function of the relative velocity of the collision partners $c_{r}$ and the temperature exponent of the coefficient of viscosity $\omega$, was formulated to correct this problem. In the VHS model the momentum cross section and the total collision cross section are again equivalent, however this leads to discrepancies in simulating flows where diffusion is important which lead to the development of the variable soft sphere (VSS) model by Koura and Matsumoto [17]. Here the momentum cross section is related to the total collision cross section by:

$$
\sigma_{M}=\frac{2}{(\alpha+1)} \sigma_{T}
$$

where $\alpha$ is the VSS scattering parameter, a function of the Schmidt number which gives the ratio of viscosity to diffusion.

The total collision cross section for the VSS model is given by [12]:

$$
\sigma_{T}=\pi d^{2} \quad \text { where } d=d_{r e f} \sqrt{\frac{\left(2 k T_{r e f} / m_{r} c_{r}^{2}\right)^{\omega-0.5}}{\Gamma(2.5-\omega)}}
$$


where $d_{\text {ref }}$ is the reference molecular diameter at temperature $T_{r e f}, k$ is Boltzmann's constant, $\Gamma$ represents the gamma function and $m_{r}$ is the reduced mass of the collision partners.

In this model a potential collision partner for the precursor molecule is generated at the collision point with components of velocity selected at random from the Maxwell-Boltzmann distribution for the local background gas conditions. The total collision cross section is then determined from equation (8) and, because the probability of a collision is a function of the total collision cross section and relative speed of the potential collision partners, the acceptance-rejection method suggested by Bird [12] is employed and, if required, a new collision partner is generated. The momentum cross section is then calculated through equation (7) and the collision position determined from the velocity of the precursor molecule and equations (2) and (3). The collision itself is then generated by random selection of appropriate deflection and azimuth angles, along with expressions for the conservation of momentum and energy. In this way the position of the collision and the collision event itself are effectively decoupled. The model assumes that the precursor concentration is sufficiently low that molecular collisions between precursor molecules are relatively infrequent.

\section{$\underline{2.3 \text { Code Implementation and Validation }}$}

The PIES model was implemented using code developed in MATLAB version 6.5. In order to test the procedures employed in the code, a series of validation studies were carried out. These were designed to test the simulated rate of diffusion of the precursor particles in the reactor and the collision procedures employed for molecular interactions.

The standard pseudorandom number generator in MATLAB "rand" was used to generate the required random variables and was started in a different state for each run. The "rand" function has been shown to have a period, which is the number of random numbers generated before any two values are repeated, of almost $2^{1430}[21]$. 


\subsubsection{Velocity Persistence in an Equilibrium Gas}

After a collision of particles of masses $m_{1}$ and $m_{2}$ in the vapour phase, the collision partners can be expected to maintain a component of their velocity in the pre-collision direction. The ratio of this post-collision component to the pre-collision velocity is known as the velocity persistence ratio which has a mean value for hard sphere particles of [19]:

$$
\varpi_{12}=\frac{1}{2} M_{1}+\frac{1}{2} M_{1}^{2} M_{2}^{-1 / 2} \ln \left[\frac{\left(M_{2}^{1 / 2}+1\right)}{M_{1}^{1 / 2}}\right] \text { where } M_{1}=\frac{m_{1}}{m_{1}+m_{2}} \& M_{1}=\frac{m_{2}}{m_{1}+m_{2}}
$$

For molecules of the same size $\varpi_{12}=0.406$, while when $m_{1}>>m_{2}, \varpi_{12} \rightarrow 1$ and the heavy particles move almost ballistically; and when $m_{1}<<m_{2}, \varpi_{12} \rightarrow 0$ and the light particles move as if they were undergoing random walk.

To validate the reactor efficiency code, the algorithm was used to calculate the persistence ratio for the movement of various noble gas particles through a uniform and stationary field of other noble gas particles. This was done by determining the magnitude of the projection of the post-collision velocity vector $\bar{v}_{2}$ onto the unit vector of the pre-collision velocity vector $\bar{v}_{1}$, and comparing this to the magnitude of the pre-collision vector $\left\|\bar{v}_{1}\right\|$. The results were averaged over a large number of collisions $N$ :

$$
\varpi_{12, \text { simulated }}=\frac{1}{N} \sum_{i=1}^{N} \frac{\bar{v}_{1} \bullet \bar{v}_{2}}{\left\|\bar{v}_{1}\right\|^{2}}
$$

The simulated results with $N=10,000$ in equation (10), utilising both hard sphere and VSS models, were compared to theoretical values obtained from equation (9) and are shown in figure 3. Because statistical error is of the order of $1 / \sqrt{N}$ the error in these calculations is approximately $1 \%$.

The hard sphere values show good agreement with the theoretical curve from equation (9) which itself assumes hard sphere collisions. This indicates that the algorithm is 
performing correctly since the persistence ratios computed by the algorithm and those from hard sphere theory are almost identical. The VSS model deviates significantly from the theoretical values especially when the ratio $m_{1} / m_{2}$ is small. This does not indicate a problem with the algorithm, but rather illustrates the limitations of the hard sphere model in real gas simulations. The VSS model can reproduce experimentally measured values of the diffusion coefficient [12] and consequently will produce more accurate values of the persistence ratio than can be produced by the hard sphere model or predicated from hard sphere theory.

\subsubsection{Comparison with Pure Random Walk}

A further check was carried out by comparing the average straight line distance travelled by small particles in a field of large particles (i.e. as $m_{1} / m_{2}$ becomes very small) to the distance predicted by pure random walk. Pure random walk occurs when the direction of movement after a collision has no relation to the pre-collision direction (i.e. $\varpi_{12}=0$ ) and corresponds to the movement of a particle undergoing surface diffusion. The average straight line distance $d$ travelled from the origin for a particle undergoing random walk is equivalent to the standard deviation of the Gaussian distribution, centred about the origin, of the distance travelled by a large number of particles $d=\lambda \sqrt{n}$, where $n$ is the number of steps taken by the particles and $\lambda$ is the mean step distance [22]. Figure 4 shows a comparison of the average straight line distance travelled by 1000 helium atoms in a uniform stationary field of xenon at $10 \mathrm{~Pa}$ (for which $\varpi_{12}=0.0159$ ) to the distance predicted by random walk, where $n$ is the number of collision events and $\lambda$ is the mean free path. Deviation between the two lines is due in part to the slight persistence of the simulated system and in the small statistical error in the simulations. 


\subsubsection{Unsteady Self Diffusion}

To validate the procedures for the VSS collision model, custom DSMC code was developed in MATLAB to measure the unsteady (i.e. time dependent) self diffusion of argon with a number density of $1.4 \times 10^{20} \mathrm{~m}^{-3}$ and a temperature of $273 \mathrm{~K}$. The analytical solution for one dimensional transient diffusion into a semi-infinite region is analogous to the transient heat conduction into a semi-infinite solid with constant surface temperature [23] such that the density of species $\mathrm{A}, \rho_{A}(x, t)$, is:

$$
\frac{\rho_{A}(x, t)}{\rho_{A, 0}}=\frac{1}{2}\left[1-\operatorname{erf}\left(\frac{x}{2 \sqrt{D_{A A} t}}\right)\right]
$$

where $\rho_{A, 0}$ is the initial density of species A, $x$ is the position from the initial separation interface of the two species, $D_{A A}$ is the self-diffusion coefficient and $\operatorname{erf}(\ldots)$ represents the error function. It should be noted that due to the requirement of species equilibrium at $x=0$, the density ratio $\rho_{A}(0, t) / \rho_{A, 0}=0.5$ for all values of time $t>0$ in a manner equivalent to the thermal equilibrium requirement of the analogous heat transfer problem.

The simulations utilised 100,000 test particles on a 1 x $0.01 \mathrm{~m}$ two dimensional grid with periodic boundary conditions in the y-direction. An ensemble average was taken to reduce statistical error in the sampling. Figure 5 compares the results of these simulations with the predicted values from equation (11) using the value for self diffusion calculated by Bird of $D_{A A}=2.95 \mathrm{~m}^{2} / \mathrm{s}[12]$. The results show agreement between the simulations and the theoretical values within the statistical scatter of the sampling and confirm the validity of the VSS collision procedures.

The results of the various verification studies confirm that the computational procedures employed in the PIES code will result in realistic diffusion rates of precursor molecules through the background flow field and gives confidence in the validity of reactor efficiency simulation results. 


\section{Investigation of Pulsed-Pressure CVD}

The PIES model was used to investigate the process of precursor diffusion from a well mixed gas to a substrate that consumes the precursor component. In order to establish the high efficiency behaviour of PP-CVD, the numerical investigation aims to determine the time scale for consumption of the precursor on the substrate. It is reasoned, that if the diffusion and deposition processes are much faster than the pump-down process, then it is possible that high precursor conversion efficiency can be achieved.

The investigation was based around noble gas molecules as a model for the precursorsolvent vapour system because these have known collision parameters and only translational degrees of freedom. The model system was necessary because very limited data is available for the collisional behaviour of the actual precursor or the solvent molecules used in typical PP-CVD processes. Of the data which is available, Tatsuda et al. estimate the hard sphere diameter of TTIP as $12 \AA$ [24] and Rubio et al. estimate the hard sphere diameter of toluene as $5.72 \AA[25]$. This data was used in the study, however the limitations of the hard sphere model have already been mentioned.

During the simulations the background gas is assumed to have no bulk velocity and to be everywhere uniform throughout the reactor volume (i.e. have no property gradients). The gas pressure throughout the reactor volume was determined at time $t$ after the start of the pump-down phase using equation (1). Although these assumptions are unphysical in a volume which is being evacuated, it will be shown that this approximation holds over the time scale required for the vast majority of precursor molecules to be consumed by the substrate. Furthermore, the rate of pump-down in this PP-CVD process is approximately $3 \mathrm{~m}^{3} / \mathrm{h}$ which translates to a bulk flow velocity of approximately $0.3 \mathrm{~m} / \mathrm{s}$ through the reactor cross section: several orders of magnitude less than the molecular velocities experienced in the flow. Flow 
visualisation experiments also confirm that there is little bulk velocity during the pump-down phase [9].

Figure 6 gives a schematic representation of the modelling process with the PP-CVD reactor geometry shown in figure 6a. As illustrated in figure $6 \mathrm{~b}$, the precursor molecule is spawned at a random point in the reactor and moves ballistically, undergoing collisions with solvent molecules, until it impacts the substrate and is deposited upon it. The pressure, and thus the number of solvent molecules, in the reactor decreases with time causing an increase in the precursor (mean) free path $\lambda$, as shown in figure $6 \mathrm{c}$. Precursor particles which strike the reactor's walls are assumed to be diffusely reflected with complete thermal accommodation. Because the reactor is axially symmetric, the computational expense of the simulations can be greatly reduced and the path of the precursor molecule can be projected onto the zero-azimuth plane.

The parameters used in the study were set to be consistent with the experimental studies of Krumdieck and Raj [8] and are summarised in table 1. 
Table 1. Simulation parameters

\begin{tabular}{lc}
\hline Reactor height (jet inlet to substrate distance), $h$ & $0.185 \mathrm{~m}$ \\
Reactor radius, $R$ & $0.03 \mathrm{~m}$ \\
Substrate radius, $r$ & $0.0225 \mathrm{~m}$ \\
Reactor peak pressure, $P_{\text {max }}$ & $1,10,100,1000 \mathrm{~Pa}$ \\
Reactor minimum pressure, $P_{\text {min }}$ & $0 \mathrm{~Pa}$ \\
Pump-down constant, $\tau_{R}$ & $2.65 \mathrm{~s}$ \\
Wall and background gas temperature, $T$ & $293 \mathrm{~K}$ \\
Noble gases studied (VSS model) & $\mathrm{He}, \mathrm{Ne}$, Ar, Kr, Xe \\
Real precursor / solvent pairs studied (HS model) & $\mathrm{TTIP} / \mathrm{Toluene}$ \\
\hline
\end{tabular}

Simulations were conducted to assess the effect of reactor peak pressure, solvent gas type and precursor molecule type on the residence time of particles within the reactor. The time taken for $95 \%$ of the precursor molecules within the reactor volume to reach the substrate $t_{95 \%}$ was chosen as a suitable measure of this residence time. Figure 7 shows the $t_{95} \%$ values for different precursor-solvent gas pairs for a reactor with $P_{\max }=10 \mathrm{~Pa}$.

The same data is presented in figure 8 showing an approximately logarithmic relationship between the precursor to solvent gas mass ratio and residence time when the data is grouped by solvent molecule type. Here $\mathrm{mr}$ is the mass of the solvent gas relative to the mass of helium. Included on this plot is the data point for the toluene-TTIP hard sphere system.

The effect of reactor peak pressure on precursor residence time was studied by simulating the pump-down phase for several precursor-solvent combinations including the hard sphere TTIP-toluene combination, Xe-Ar (which has a similar precursor to solvent mass ratio as TTIP-toluene) and, as a point of comparison, Ar-He. Simulations we not carried out 
for peak pressures exceeding $1000 \mathrm{~Pa}$ due to the large computational expense required. The larger magnitude of the error on the higher pressure values is a consequence of a smaller sample size due to the large computational expense. The results of this study are illustrated in figure 9 .

\section{Results and Discussion}

The results in the previous section can be compared to reactor pump-down time $t_{P}$ to gain an appreciation of the processes leading to the observed high precursor conversion efficiencies in PP-CVD. Typically, PP-CVD reactors operate with a pulse period $t_{P} \geq 4 \tau$ and $t_{P}>t_{i}$ to ensure that both mass flux uniformity is high after the injection phase and the reactor pressure does not climb over a period of several pulses [26]. For the reactor studied in these simulations the reactor pump-down time $t_{P}=10.6$ seconds.

From figures 7 and 8 we see that in every case residence time $t_{95 \%}<<10.6$ s for $P_{\max }=10 \mathrm{~Pa}$. Because the time for diffusion of precursor particles to the substrate is much less than the pump-down time we can expect very high efficiencies for a reactor operating in this regime. The relationship between the different precursor-solvent combinations in these figures reveals that the mass ratio of the precursor to the solvent along with the relative mass of the solvent both have large effects on the precursor residence time. Lighter solvent molecules result in a decrease in precursor residence time as does a decrease in precursor size relative to the size of the solvent.

The reasons for these relationships can be readily understood by examining equation (2). As the collision cross section of the collision partners increases, the free-path of the molecules within the gas decreases. Larger molecules undergo more scattering events and, coupled with the fact that their molecular speed is lower than smaller molecules at the same temperature, their residence time increases, as would be expected from diffusion 
relationships. Clearly the lesson here is that solvents should be chosen with as low a molecular weight as possible for the PP-CVD process. Similar results have been reported for Jet Vapour Deposition (JVD), in which a helium carrier gas stream is employed to deposit gold vapour [27], and in Directed Vapour Deposition (DVD) [16].

From figure 9 it can be seen that the arrival rate for the precursor at the substrate is highly dependent on reactor peak pressure. As $P_{\max }$ increases so that the residence time $t_{95 \%}$ approaches $t_{P}$ we can expect a corresponding drop in reactor efficiency. This result is as would be expected from diffusion relationships, however it should be kept in mind that the decreasing reactor pressure throughout the pump-down phase given by equation (1) means that precursor residence time will be significantly lower than for a reactor which operates at steady pressures.

It has been shown that when the precursor residence time $t_{95 \%}<<t_{P}$ we can expect high reactor efficiencies. When this condition is satisfied, convective effects within the reactor due to bulk flow are minimal and precursor particles diffuse to the surface due to random molecular walk. Random walk is a very rapid process in stationary gases at the relatively low pressures in the pump-down phase of PP-CVD and consequently high precursor conversion efficiencies are achieved. In steady flow reactors, or in unsteady reactors with a short pumpdown time, convective effects are high and precursor particles must diffuse in a direction which is generally normal to the bulk flow direction in order to reach the deposition surface. Consequently convective effects move large quantities of the precursor out of the reactor before the molecules can diffuse to the deposition surface resulting in low precursor conversion efficiencies.

The study confirms that the physical mechanisms for high efficiencies in PP-CVD reactors occur when the rate of precursor diffusion, adsorption onto the substrate and thermal decomposition are faster than the reactor pump-down rate. The study also shows that to 
achieve high efficiencies solvents of minimal molecular size should be employed and confirms that high reactor peak pressures will result in a decrease in precursor conversion efficiency.

The intend to extend the current study by generating the solvent gas flow field during both the pump-down and injection phases using either pure DSMC or hybrid continuumDSMC methods, and then using the PIES method to track precursor particles though the flow field. This step will help confirm the validity of the minimal bulk flow assumption and will allow the development of the uniform mass flux field to be studied in detail.

\section{Conclusion}

The development of a model for the pump-down phase in PP-CVD has allowed the physical mechanisms for the high precursor conversion efficiencies in this process to be studied in detail. The model utilises the hard sphere and variable soft sphere (VSS) collision models to track individual precursor particles through a uniform expanding solvent flow field within the reactor volume. The validity of the model was confirmed by studying the persistence ratio in collisions between hard sphere molecules of different sizes and the transient self-diffusion behaviour of VSS argon molecules.

In the PP-CVD process, when the time taken for the majority of precursor molecules to reach the substrate is much less than the reactor pump-down time, high reactor efficiencies can be expected. This is because the random drift of precursor to the substrate takes much less time than for molecules to be removed from the reactor by the vacuum pump. The relationship between precursor and solvent size has also been studied confirming that larger precursor molecules will result in a decrease in reactor efficiency as will heavier solvents.

The simulations show that the efficiency results from experimental deposition studies are plausible and provide a valuable step in understanding the complex unsteady flow field 
exhibited in the PP-CVD process. The present authors intend to extend the present study by using the precursor tracking model to investigate the movement of precursor through a solvent flow field generated using DSMC or hybrid continuum-DSMC methods.

\section{References}

[1] M. Ohring, Materials Science of Thin Films, Academic Press, San Diego (CA), (2002).

[2] V. Versteeg, C.T. Avedisian, R. Raj, U.S. Patent No.: 5,541,260 (1994).

[3] S. Krumdieck, R. Raj, Conversion Efficiency of Alkoxide Precursor to Oxide Films Grown by an Ultrasonic-Assisted Pulsed Liquid Injection Metalorganic Chemical Vapor Deposition (Pulsed-CVD) Process, J. Am. Ceram. Soc. 82 (1999) 1605-1607.

[4] S. Krumdieck, O. Sbaizero, A. Bullert, R. Raj, Solid Yttria-Stabilized Zirconia Films by Pulsed Chemical Vapor Deposition from Metal-organic Precursors, J. Am. Ceram. Soc. 85 (2002) 2873-2875.

[5] S. Krumdieck, O. Sbaizero, A. Bullert, R. Raj, YSZ layers by pulsed-MOCVD on solid oxide fuel cell electrodes, Surf. Coat. Technol. 167 (2003) 226-233.

[6] S. Krumdieck, R. Raj, Experimental Characterization and Modelling of Pulsed MOCVD with Ultrasonic Atomization of Liquid Precursor, Chem. Vap. Deposition 7 (2001) 85-90.

[7] S. Krumdieck, Kinetic Model of Low Pressure Film Deposition from Single Precursor Vapor in a Well-Mixed, Cold-Wall Reactor, Acta mater. 49 (2001) 583-588.

[8] S. Krumdieck, R. Raj, Growth rate and morphology for ceramic films by pulsed-MOCVD, Surf. Coat. Technol. 141 (2001) 7-14.

[9] S. Krumdieck, H.M. Cave, A. Peled, S. Baluti, R. Albert, M. Jermy, J.-Y. Lee, Expansion transport regime in pulsed-pressure chemical vapor deposition, submitted to Vacuum (2006). [10] H.M. Cave, S. Krumdieck, M. Jermy, CFD prediction of continuum breakdown in an unsteady under-expanded jet, in review, Vacuum (2005). 
[11] G.E. M. Karniadakis, A. Beskok, Micro flows: fundamentals and simulation, SpringerVerlag, New York (NY) (2002).

[12] G.A. Bird, Molecular Gas Dynamics and the Direct Simulation of Gas Flows, Oxford University Press, Oxford (1994).

[13] G.J. LeBeau, and F.E. Lumpkin, Application highlights of the DSMC Analysis Code (DAC) software for simulating rarefied flows, Comput. Methods Appl. Mech. Eng. 191 (2001) 595-609.

[14] I.D. Boyd, Y. Jafry, J.V. Beukel, Particle Simulations of Helium Microthruster Flows, J. Spacecraft Rockets 31 (1994) 271.

[15] H.A. Al-Mohssen, N.G. Hadjiconstantinou, Arbitrary-pressure chemical vapor deposition modeling using direct simulation Monte Carlo with nonlinear surface chemistry, J. Comput. Phys. 198 (2004) 617-627.

[16] J. F. Groves, PhD Thesis, University of Virginia (1998).

[17] K. Koura, and H. Matsumoto, Variable soft sphere molecular model for inverse power law or Lennard-Jones potential, Phys. Fluids A 3 (1991) 2459-2465.

[18] E.W. McDaniel, Collision Phenomena in Ionized Gases, John Wiley \& Sons, New York (NY) (1964).

[19] S. Chapman, T.G. Cowling, The Mathematical Theory of Non-uniform Gases, Cambridge University Press, Cambridge (1970).

[20] G.A. Bird, Monte Carlo simulation in an engineering context, AIAA International Symposium on Rarefied Gas Dynamics, Charlottesville (VA) (1981).

[21] C.B. Moler, Random Thoughts: $10^{435}$ years is a very long time, Matlab News \& Notes (Fall, 1995).

[22] D.L. Smith, Thin-Film Deposition: Principles and Practice, McGraw-Hill, Boston (MA) (1995). 
[23] F.P. Incropera, D.P. DeWitt, Fundamentals of Heat and Mass Transfer, 5th ed., John Wiley \& Sons, New York (NY) (2002).

[24] N. Tatsuda, Y. Fukushima, H. Wakayama, Penetration of Titanium Tetraisopropoxide into Mesoporous Silica Using Supercritical Carbon Dioxide, Chem. Mater. 16 (2004) 17991805.

[25] J.E.F. Rubio, V.G. Baonza, M. Taravillo, J. Nunez, M. Caceres, A dynamic light scattering study of the hypersonic relaxation in liquid toluene, J. Chem. Phys. 115 (2001) $4681-4688$

[26] S. Krumdieck, J.-Y. Lee, H. Raatz, Uniform Molecular Flux in a Vertical Reactor with Pulsed Transition Regime Gas Flow, Proc. - Electrochem. Soc. 8 (2003) 179-185.

[27] B.L. Halpern, J.J. Schmidt, Multiple jets and moving substrates: Jet Vapor Deposition of multicomponent thin films, J. Vac. Sci. Technol. A. 12(4) (1994) 1623. 


\section{List of Figures}

Figure 1. Functional schematic of a Pulsed Pressure Chemical Vapour Deposition (PP-CVD) reactor with a plot of the reactor pressure during operation.

Figure 2. "PIES" reactor efficiency model computational flow diagram.

Figure 3. Code validation study comparing molecular persistence ratio predicted using the hard sphere and VSS collision models to theoretical values.

Figure 4. Code validation study comparing the average straight line distance travelled by helium atoms in a stationary uniform field of xenon molecules at $10 \mathrm{~Pa}$.

Figure 5. Validations of VSS collision procedure by DSMC simulations of transient selfdiffusion of argon.

Figure 6. a) PP-CVD reactor geometry showing zero-azimuth axisymmetric plane and precursor particle tracking schematic at b) time $t_{1}$ and c) time $t_{2}$ where $t_{2}>t_{1}$.

Figure 7. Precursor residence time $t_{95 \%}$ for different precursor-solvent combinations with $P_{\max }$ $=10 \mathrm{~Pa}$.

Figure 8. Precursor residence time $t_{95} \%$ for different precursor-solvent combinations with $P_{\max }$ $=10 \mathrm{~Pa}($ logarithmic scale). 
Figure 9. Precursor residence time $t_{95 \%}$ for different reactor peak pressures $P_{\max }$ (logarithmic scale). 
Figures

Figure 1.
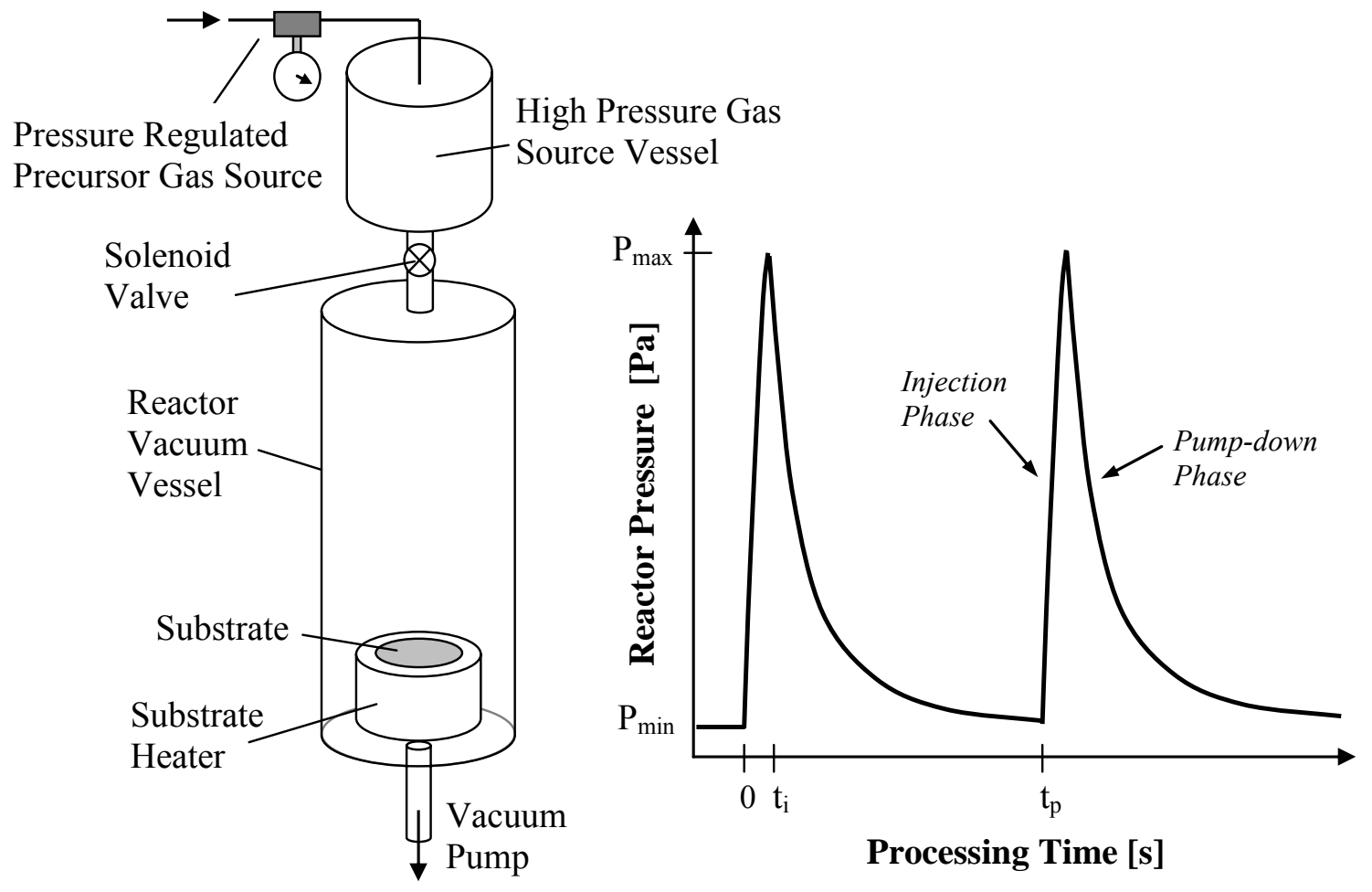
Figure 2.

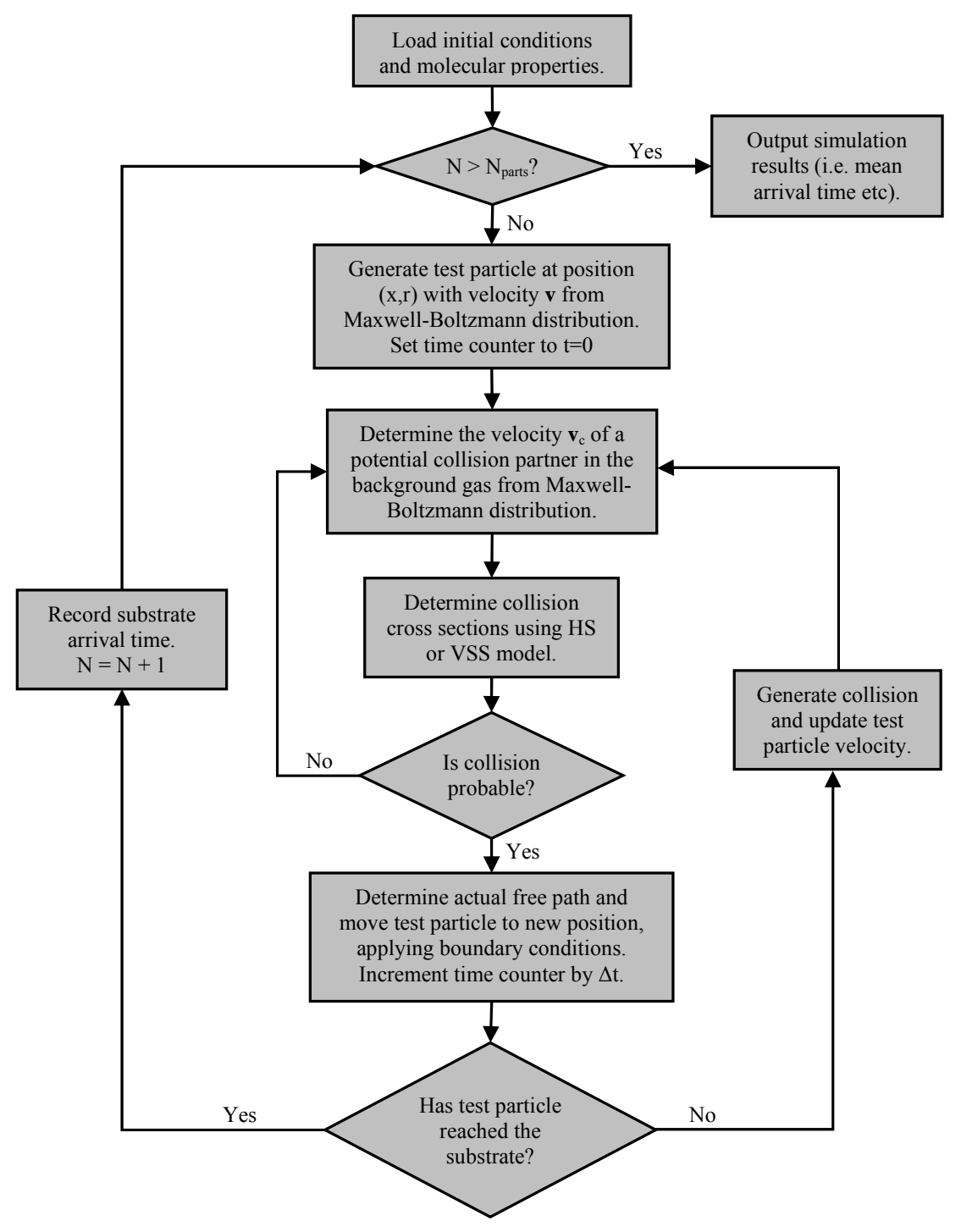


Figure 3.

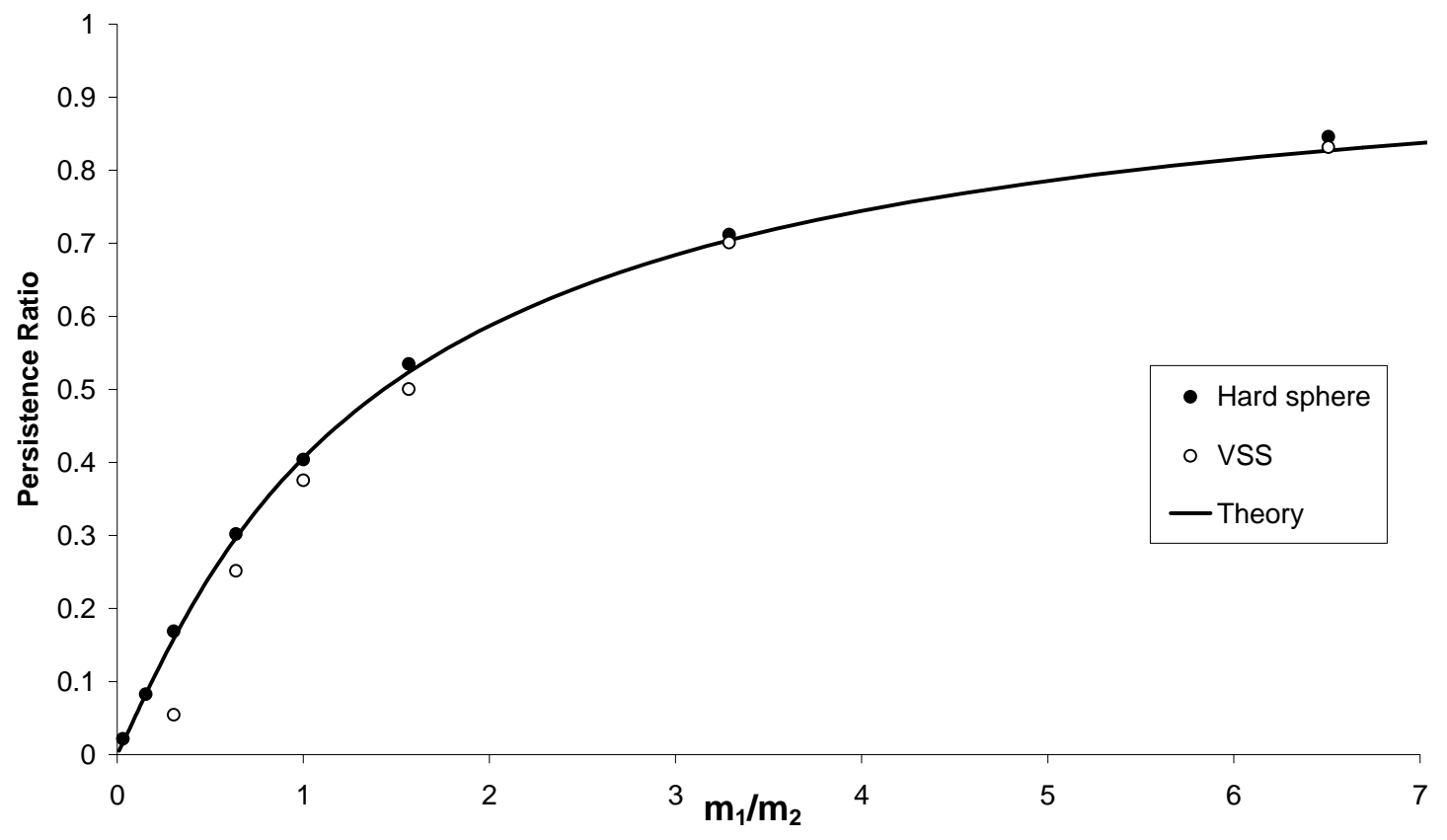


Figure 4.

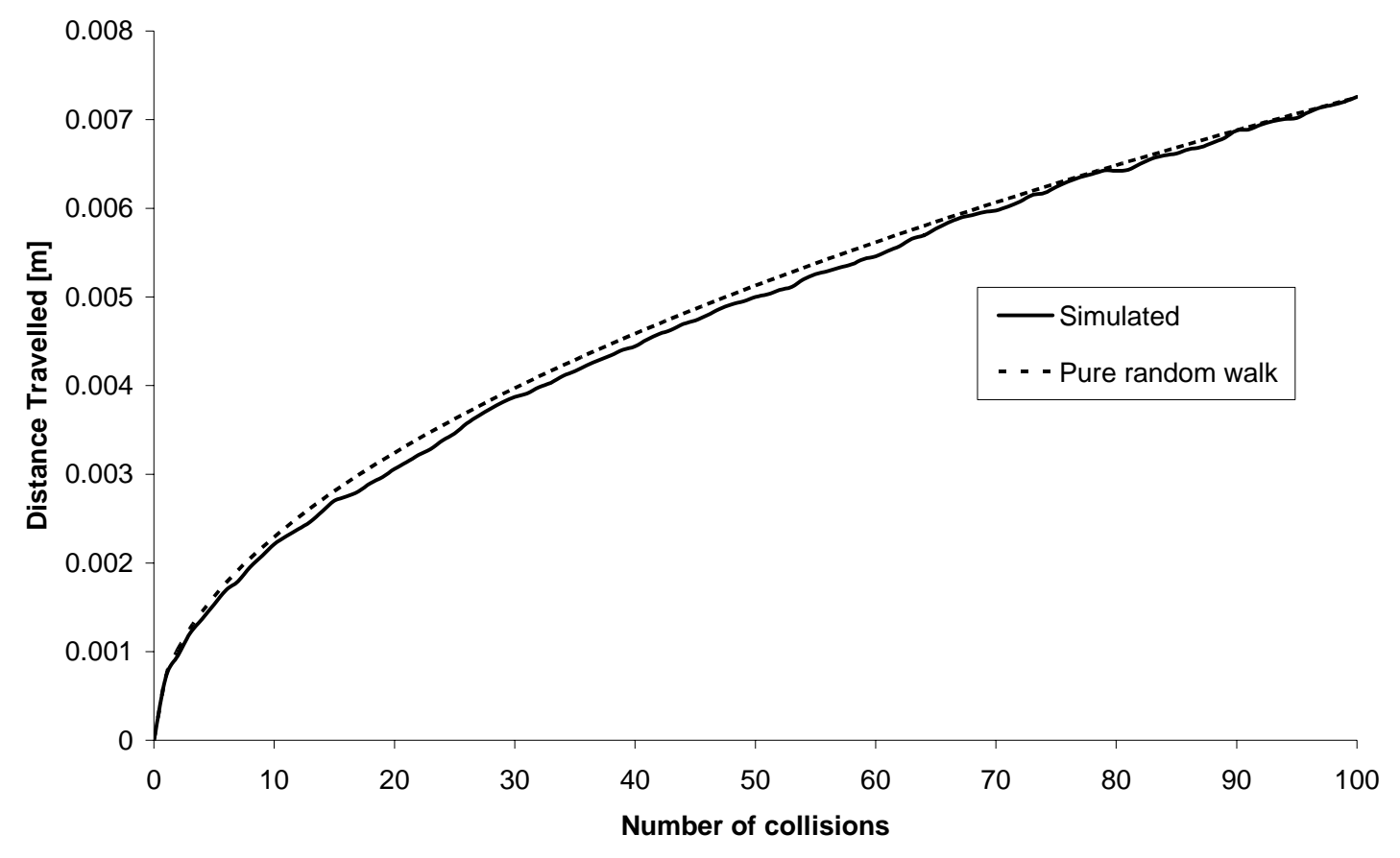


Figure 5.

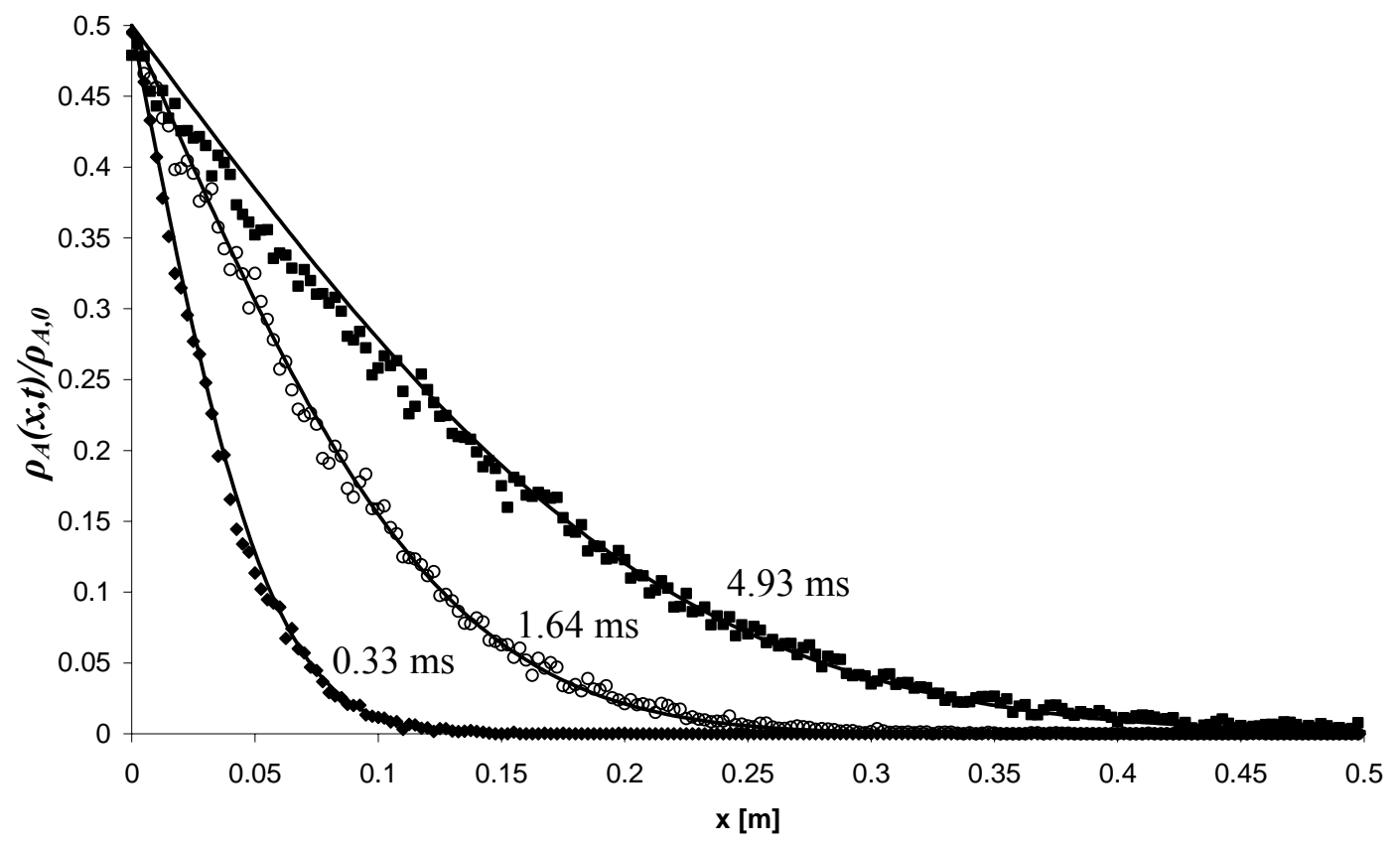


Figure 6.

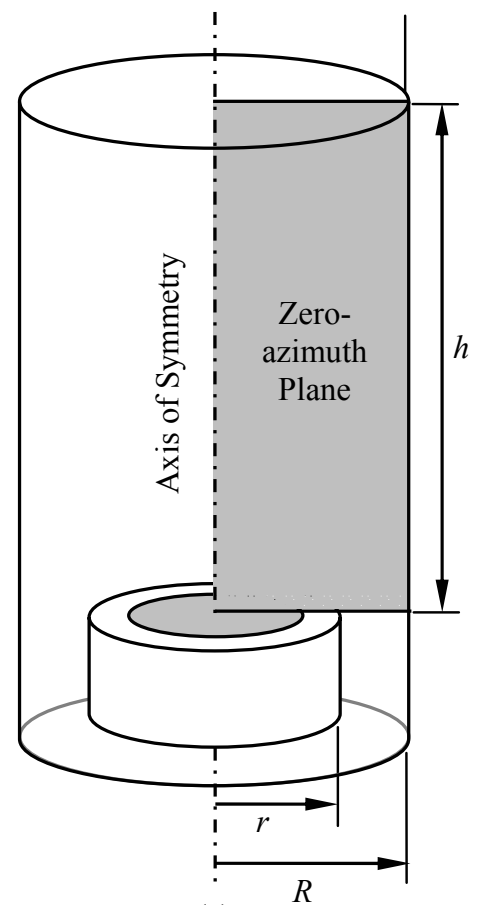

(a)

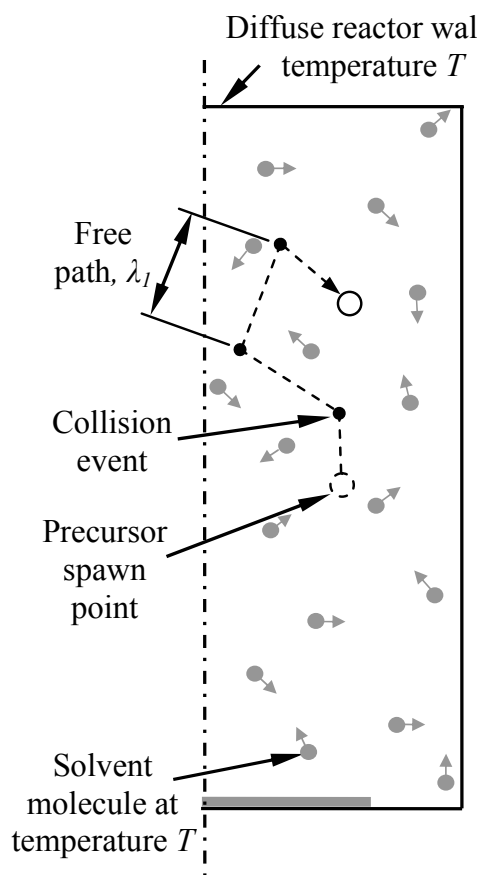

(b)

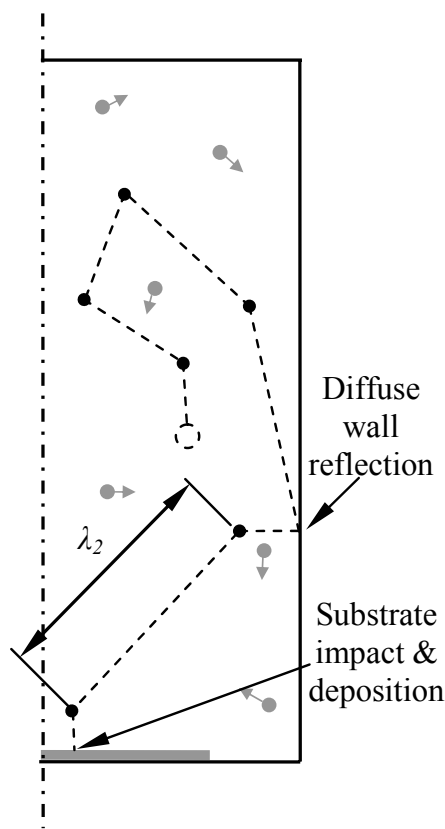

(c) 
Figure 7.

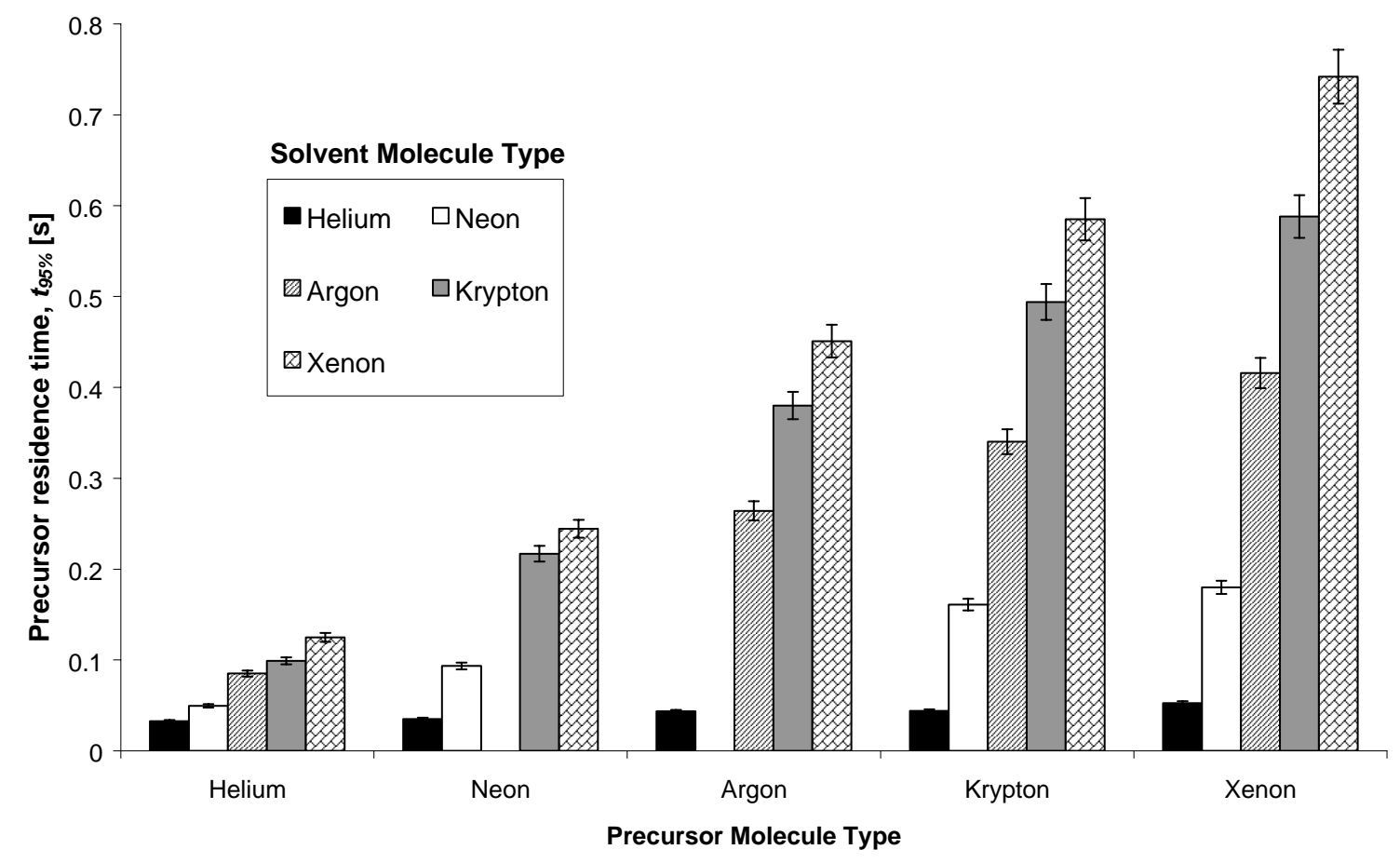


Figure 8.

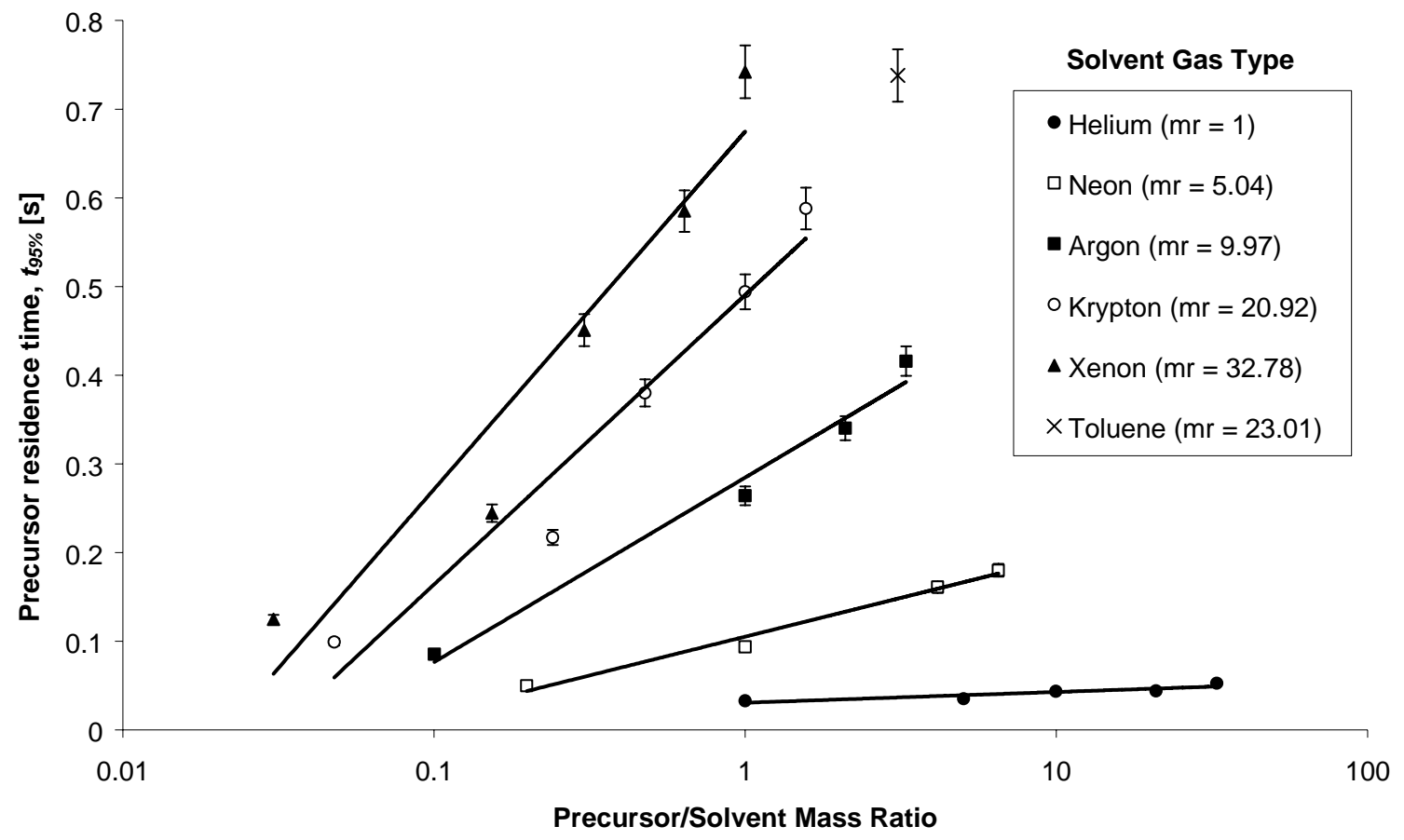


Figure 9.

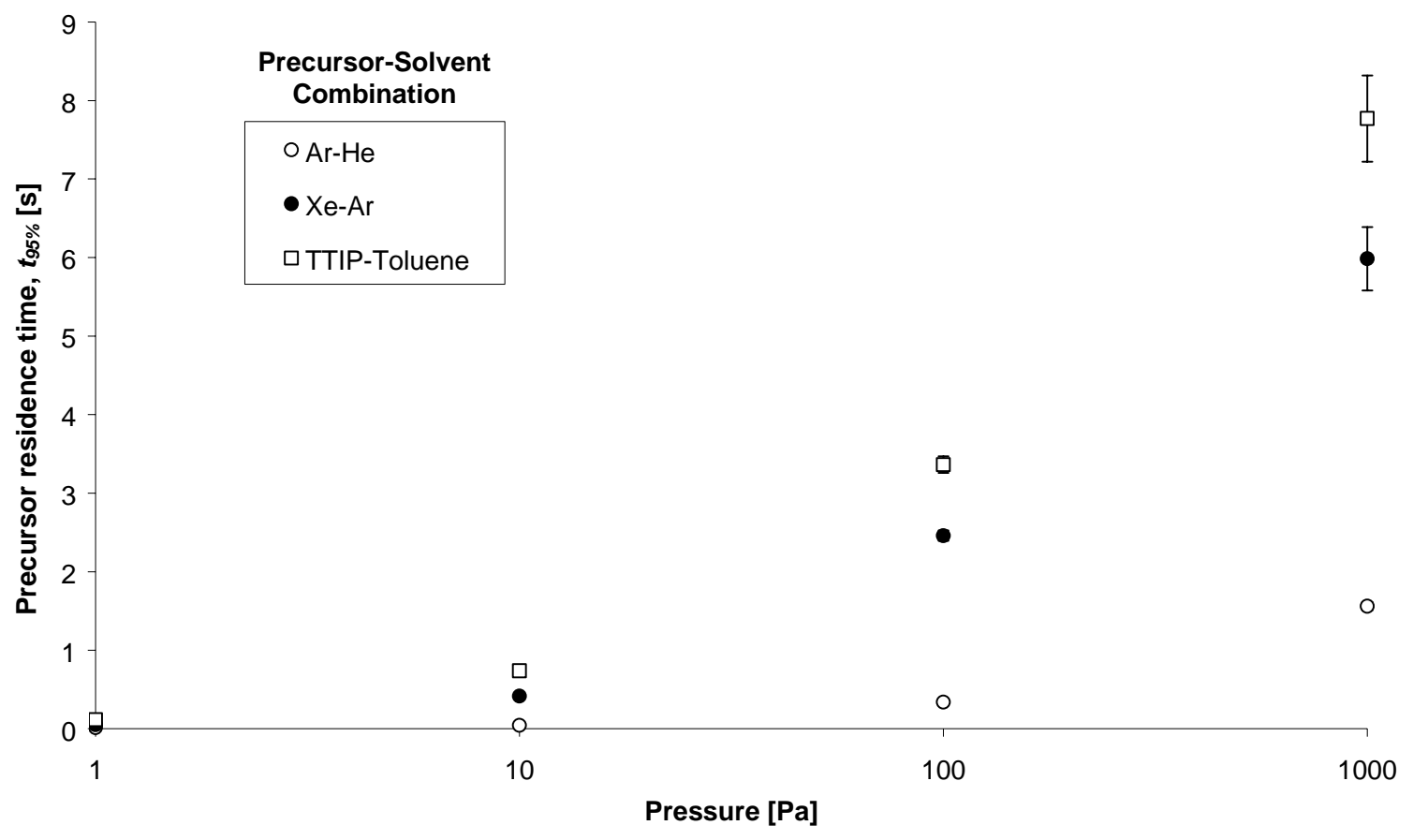

